\title{
Functional analysis of filipin tailoring genes from Streptomyces filipinensis reveals alternative routes in filipin III biosynthesis and yields bioactive derivatives
}

Tamara D Payero ${ }^{1,2}$, Cláudia M Vicente ${ }^{1,2}$, Ángel Rumbero³, Eva G Barreales ${ }^{1}$, Javier Santos-Aberturas²,4, Antonio de Pedro ${ }^{1}$ and Jesús F Aparicio ${ }^{1 *}$

\begin{abstract}
Background: Streptomyces filipinensis is the industrial producer of filipin, a pentaene macrolide, archetype of nonglycosylated polyenes, and widely used for the detection and the quantitation of cholesterol in biological membranes and as a tool for the diagnosis of Niemann-Pick type $C$ disease. Genetic manipulations of polyene biosynthetic pathways have proven useful for the discovery of products with improved properties. Here, we describe the late biosynthetic steps for filipin III biosynthesis and strategies for the generation of bioactive filipin III derivatives at high yield.

Results: A region of 13,778 base pairs of DNA from the S. filipinensis genome was isolated, sequenced, and characterized. Nine complete genes and two truncated ORFs were located. Disruption of genes proved that this genomic region is part of the biosynthetic cluster for the 28-membered ring of the polyene macrolide filipin. This set of genes includes two cytochrome P450 monooxygenase encoding genes, filC and filD, which are proposed to catalyse specific hydroxylations of the macrolide ring at C26 and $\mathrm{C}^{\prime}$ ' respectively. Gene deletion and complementation experiments provided evidence for their role during filipin III biosynthesis. Filipin III derivatives were accumulated by the recombinant mutants at high yield. These have been characterized by mass spectrometry and nuclear magnetic resonance following high-performance liquid chromatography purification thus revealing the post-polyketide steps during polyene biosynthesis. Two alternative routes lead to the formation of filipin III from the initial product of polyketide synthase chain assembly and cyclization filipin I, one trough filipin II, and the other one trough 1'-hydroxyflipin I, all filipin III intermediates being biologically active. Moreover, minimal inhibitory concentration values against Candida utilis and Saccharomyces cerevisiae were obtained for all filipin derivatives, finding that 1'-hydroxyfilipin and especially filipin II show remarkably enhanced antifungal bioactivity. Complete nuclear magnetic resonance assignments have been obtained for the first time for 1'-hydroxyfilipin I.
\end{abstract}

Conclusions: This report reveals the existence of two alternative routes for filipin III formation and opens new possibilities for the generation of biologically active filipin derivatives at high yield and with improved properties.

Keywords: Antifungal agent, Cytochrome P450 monooxygenase, Filipin biosynthesis, Filipin derivatives, Polyene macrolide, Streptomyces

\footnotetext{
*Correspondence: jesus.aparicio@unileon.es

${ }^{1}$ Area of Microbiology, Faculty of Biology, Universidad de León,

24071 León, Spain

Full list of author information is available at the end of the article
} 


\section{Background}

Filipin is a 28-membered ring pentaene macrolide antifungal antibiotic produced by S. filipinensis, S. avermitilis, and $S$. miharaensis [1-3]. Unlike the majority of polyenes, it is devoid of sugar, and constitutes the archetype of non-glycosylated polyenes. However, it also interacts with membrane sterols causing the alteration of membrane structure [4]. Most polyene macrolides display significantly higher affinity for ergosterol (the main sterol in fungal membranes) than for cholesterol-containing membranes (the sterol in mammalian cells), and this is thought to be the reason for the selective toxicity of these molecules, but filipin shows a similar affinity for both sterols. This property makes it useless for its application in human therapy due to its toxic side effects, but has permitted its application as a tool for the diagnosis of Niemann-Pick type $\mathrm{C}$ disease, a characteristic cholesterol overloaded lysosomal disorder of genetic origin [5], and as a probe for the detection and quantification of cholesterol in cellular membranes [6]. As other macrocyclic polyketides, filipin is synthesized by the action of type I modular polyketide synthases. Its biosynthetic gene cluster (pte) has been identified in the avermectinproducing S. avermitilis NRRL 8165 upon sequencing of its genome and encodes 14 polyketide synthase modules within five multifunctional enzymes, and eight additional proteins that presumably govern modification of the polyketide skeleton and regulation of gene expression [7-9].

The post-polyketide synthase biosynthetic tailoring of polyene macrolides usually involves oxidations catalysed by cytochrome P450 monooxygenases (P450s or CYPs). Members from this class of enzymes are common in macrolide biosynthetic gene clusters, and their specificities vary considerably toward the substrates utilised and the positions of the hydroxyl functions introduced. In nature, filipin is produced as a mixture of related compounds known as the filipin complex (filipins I-IV) [10], being filipin III the major component. These components vary in the number of post-polyketide hydroxyl functions introduced, thus filipin I has two hydroxyl groups fewer than filipin III, filipin II is filipin I hydroxylated at C26 [11] and filipin III the result of filipin II hydroxylation at $\mathrm{C1}^{\prime}$, while filipin IV is thought to be an epimer of filipin III [12]. Of these compounds, only filipin III has been structurally characterised [13].

Two cytochrome P450 monooxygenases have been proposed to be responsible for the variability of the filipin complex in S. avermitilis, CYP105P1 (PteC) and CYP105D6 (PteD), and their crystal structures and in vitro catalytic activities have been determined [14]. Although their role in vivo remains uncharacterized, CYP105P1 and CYP105D6 catalyze filipin hydroxylation in vitro at $\mathrm{C} 26$ and $\mathrm{C} 1^{\prime}$ respectively [14].
In order to gain insight into the mechanism of filipin III formation we set out to get gene deletion mutants of both cytochrome P450 monooxygenases, but S. avermitilis turned out to be a very low producer [9], hence we decided to investigate filipin biosynthesis in S. filipinensis, a strain that produces 1,000-fold more. Interestingly, we found that two alternative routes lead to the formation of filipin III from the product of polyketide synthase assembly and cyclisation filipin I.

\section{Results}

\section{Identification and cloning of filipin tailoring genes}

The filipin biosynthetic genes were identified by hybridization using a probe obtained by PCR amplification of S. filipinensis chromosomal DNA with oligonucleotides derived from conserved stretches of the filipin PAS-LuxR regulator pteF from S. avermitilis (see "Methods"). A cosmid library was constructed and a total of $13,778 \mathrm{bp}$ of contiguous DNA was cloned. All the cosmids were mapped with restriction enzymes NotI, BamHI and $E c o$ RI. Internal NotI fragments were the same size as their homologous fragments of S. filipinensis total DNA, suggesting that the cloned DNA was not rearranged.

Once a gene homologous to $p t e F$ was identified (filF), the remaining genes of the subcluster were identified by chromosome walking. The deduced gene organization within this region is shown in Fig. 1.

\section{In silico analysis and arrangement of genes}

Computer-assisted analysis of the sequenced region revealed nine complete genes, and two truncated open reading frames (ORFs). All these genes, but one (see below), showed a good synteny with the homologous pte genes from the filipin cluster of S. avermitilis [8], and following their nomenclature they were named filB, filC, filD, filE, filR, filF, filG, and filH. The degree of identity among coding regions ranged between 87 and 96\% (at the protein level; Fig. 1) with a degree of divergence around $10 \%$, whereas among intergenic regions such variability was much higher reaching a $40 \%$ divergence, including nucleotide deletions, substitutions, and insertions. Some intergenic regions were shorter in S. filipinensis (Fig. 1). For instance, in the region between filC and filD a $526 \mathrm{nt}$ deletion occurred, while in the region between filF and filG there is a deletion of $116 \mathrm{nt}$ (Fig. 1). Changes in the intergenic regions could potentially have effects on the gene transcription given that these regions may include promoter activities (see below).

Upstream from this set of genes (Fig. 1) there is an incomplete ORF whose deduced product showed high identity with PteA5 from $S$. avermitilis, the last polyketide synthase during filipin biosynthesis [2]. The product of the gene located downstream, filB, is a putative 


\begin{tabular}{|c|c|c|c|c|c|c|c|}
\hline \multirow[b]{2}{*}{ Protein } & \multicolumn{3}{|c|}{ S. filipinensis } & \multicolumn{3}{|c|}{ S. avermitilis } & \multirow[b]{2}{*}{$\begin{array}{c}\text { Protein identity } \\
\%\end{array}$} \\
\hline & Proposed function & Aminoacids & $\begin{array}{l}\text { upstream intergenic } \\
\text { region (nt) }\end{array}$ & Protein & Aminoacids & $\begin{array}{l}\text { upstream intergenic } \\
\text { region (nt) }\end{array}$ & \\
\hline FilB & crotonyl-CoA reductase & 416 & 60 & PteB & 418 & 60 & 94 \\
\hline FilC & P450 monooxygenase & 399 & 47 & PteC & 399 & 46 & 96 \\
\hline FilD & P450 monooxygenase & 404 & 55 & PteD & 404 & 581 & 91 \\
\hline FilE & ferredoxin & 64 & 44 & PteE & 64 & 44 & 92 \\
\hline FilR & SARP-LAL regulator & 1190 & 48 & PteR & 1197 & 55 & 88 \\
\hline FilF & PAS-LuxR regulator & 192 & 406 & PteF & 192 & 399 & 94 \\
\hline FilG & cholesterol oxidase & 547 & 349 & PteG & 547 & 465 & 94 \\
\hline FilH & thioesterase & 255 & 88 & PteH & 255 & 96 & 89 \\
\hline FilI & $\begin{array}{l}\text { PadR family } \\
\text { transcriptional regulator }\end{array}$ & 190 & 127 & - & - & - & - \\
\hline
\end{tabular}

Fig. 1 Comparison of filipin tailoring gene clusters from S. filipinensis and S. avermitilis. The size of the upstream region of each gene is indicated in nucleotides. Arrows indicate the organization of transcriptional units. The transcriptional organization of S. avermitilis has been included for comparison [9].

2-octenoyl-CoA reductase that may provide the hexylmalonyl-CoA needed for the last polyketide chain extension during filipin biosynthesis [15]. The two genes that follow ( filC and filD) encode cytochrome P450 monooxygenases (399 and 404 amino acids respectively) that by analogy with their counterparts from $S$. avermitilis are presumed to be responsible for hydroxylations at $\mathrm{C} 26$ and $\mathrm{C}^{\prime}{ }^{\prime}$ [14]. The filE gene immediately downstream from filD (Fig. 1) encodes a small acidic protein (64 amino acids) with convincing similarity to ferredoxins.

Convergent to filE lays filR. FilR $(1,190 \mathrm{aa})$ is orthologous to the transcriptional activator of pimaricin biosynthesis PimR, an archetype of a particular class of regulators that combine an N-terminal DNA-binding domain corresponding to the SARP family of transcriptional activators with a C-terminal half homologous to guanylate cyclases and LAL regulators (Large ATP-binding regulators of the LuxR family) [16]. Recently we have characterized PimR mode of action, and determined that it binds an operator that contains three heptameric direct repeats of the consensus CGGCAAG with 4 bp spacers upstream from cognate promoters [17]. Divergently transcribed from filR is filF, a regulatory gene encoding a PAS-LuxR regulator (192 amino acids). These type of regulators are characteristic of polyene gene clusters [18], and have been demonstrated to be involved in the direct activation of given promoters within the cluster [19]. Recently, we have characterized PteF mode of action and determined that it activates transcription from the promoters of polyketide synthase genes $p t e A 1$ and pteA2 directly [9], and cross-regulates other clusters, e.g. the one involved in oligomycin biosynthesis [20].

The cluster also contains a cholesterol oxidase encoding gene, filG (Fig. 1), whose product has been proposed to act as antifungal sensor in S. natalensis [21], and a gene, $\mathrm{filH}$, whose product resembles discrete thioesterases involved in polyketide proofreading [22, 23].

Downstream from $\mathrm{filH}$, there is an ORF that is absent in $S$. avermitilis which shows a putative dipeptidase gene (SAV 7575) instead. Its product (FilI) showed convincing similarity to PadR transcriptional regulators (Pfam PF03551), a class of regulators that control diverse processes including multi-drug resistance, virulence and detoxification, and secondary metabolite biosynthesis [24-26]. To ascertain that this gene belonged to the filipin cluster, we inactivated it by means of PCR targeting (see "Methods"). The fermentation broth, produced by double cross-over mutants, was extracted with ethyl acetate and analysed for the presence of filipin III (the major component of the filipin complex). HPLC assays indicated that filipin III production by the mutant strain was severely increased when compared with the parental strain, while showing a similar growth profile (Fig. 2). S. filipinensis $\Delta$ fill produced about double of the filipin III accumulated by the wildtype strain at $72 \mathrm{~h}(280 \mathrm{mg} / \mathrm{l})$ (Fig. 2). This result indicates that fil belongs to the cluster and that its product Fill must be a negative regulator of filipin biosynthesis 
a

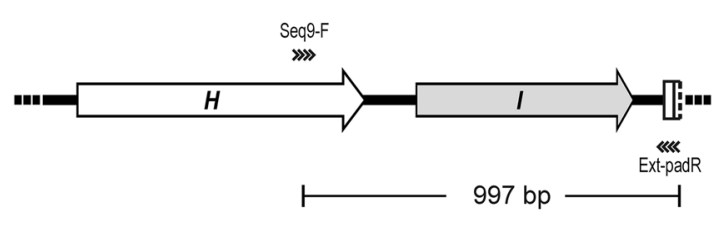

S. filipinensis WT

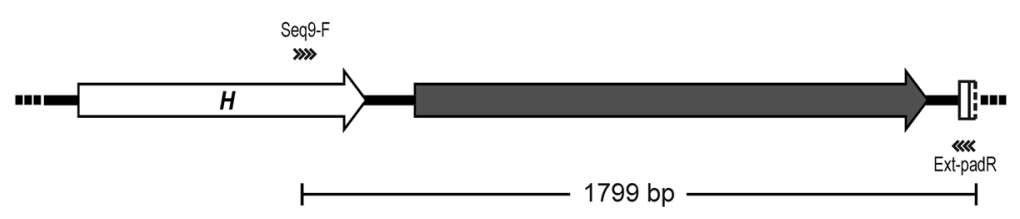

b

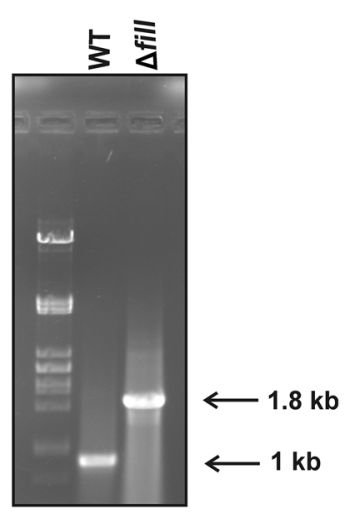

S. filipinensis $\Delta$ fill
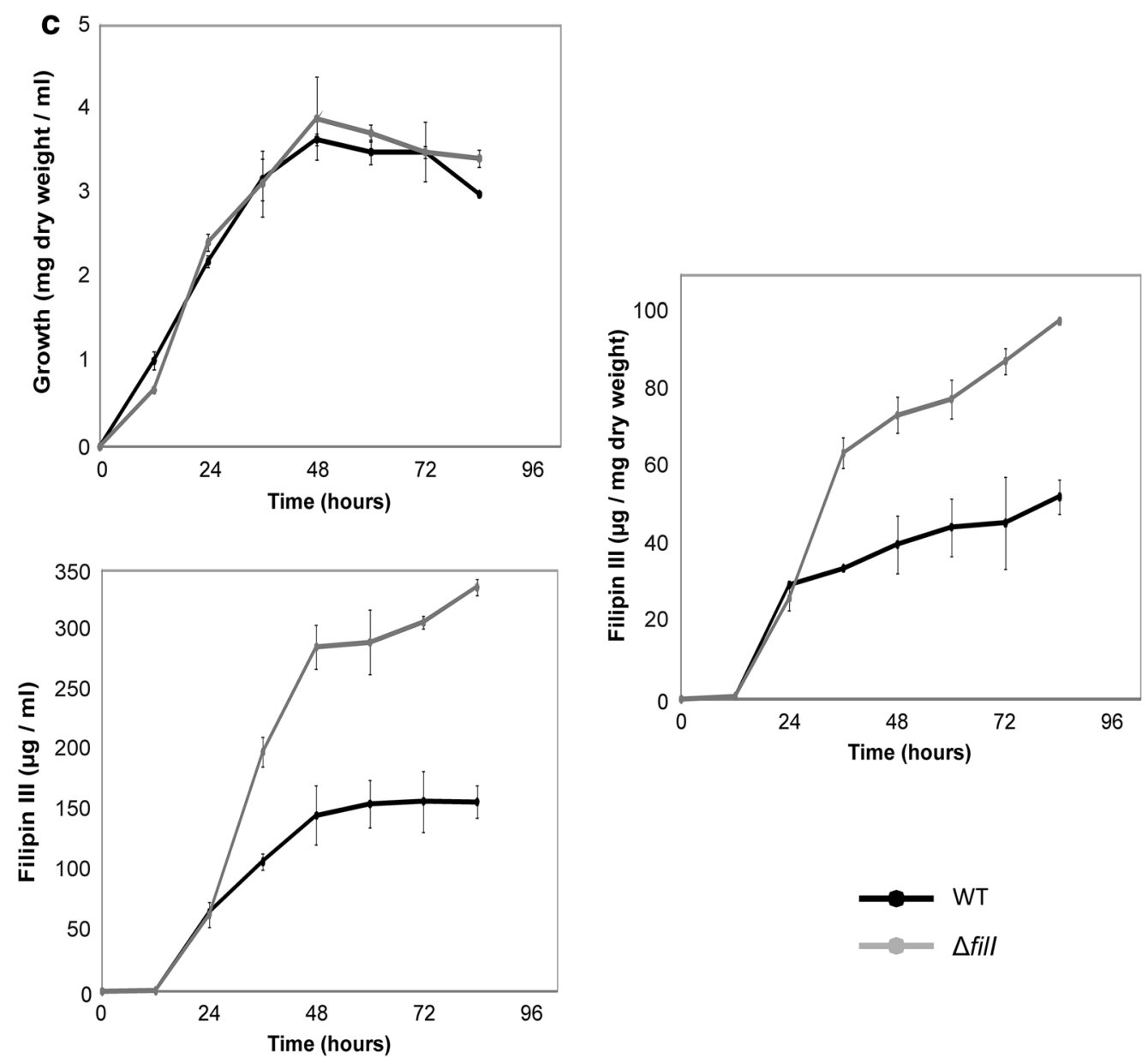

Fig. 2 Fill inactivation increases filipin III production. a Predicted PCR fragment amplification of the parental strain and the mutant. The primers used in the assay are indicated with arrowheads. The acc(3)IV-oriT cassette is indicated in black. b PCR analysis of the wild type and the mutant. $\mathbf{c}$ Growth curves and time course quantification of the filipin III production in S. filipinensis (black) and S. filipinensis $\Delta$ fill (grey).

(Fig. 2). Flanking the right end of the cluster, and downstream from fill, there is an incomplete ORF whose partial product showed convincing similarity to acyl-CoA dehydrogenases.

\section{Organization of fil transcriptional units}

In order to define an overall picture of the transcriptional arrangement of the fil genes, an accurate identification of operons was needed. We thus decided to analyse 
the possible co-transcription of neighbouring genes by reverse transcriptase-polymerase chain reaction experiments. Total RNA was prepared from S. filipinensis after growth for $48 \mathrm{~h}$. Primers were designed to obtain cDNAs corresponding to unabated transcription between two genes (Additional file 1: Table S1). Transcripts were analysed after 40 PCR cycles to ensure that even low-level transcribed genes were detected. A primer pair designed to amplify a cDNA of the $r r n A 1$ gene was used as an internal control. These analyses were carried out at least three times for each primer pair. Following this strategy, filA5, filB, filC, filD, and filE could be co-transcribed since unabated transcription was observed between the upstream and the downstream gene. Similarly filG, filH and fill could also be co-transcribed. No transcripts were detected connecting filF and filG, thus suggesting that filG should have its own promoter. The genes filR and filF must also have their own promoters as can be deduced from their chromosomal arrangement in a divergent manner. Figure 1 shows the deduced organization of transcriptional units.

\section{Functional analyses of P450 monooxygenases}

In order to determine the function of filC, and filD, we inactivated them by using the REDIRECT gene replacement technology as indicated in "Methods". Double-crossover mutants were screened by apramycin resistance. These were verified by PCR analysis (Fig. 3). Additionally, the double mutant S. filipinensis $\triangle$ filCD was also constructed using the same methodology.

The new S. filipinensis $\triangle$ filC, $\triangle$ filD, and $\triangle$ filCD strains had growth and morphological characteristics identical to those of S. filipinensis wild type when grown on solid or liquid media, suggesting that those genes had no role in bacterial growth or differentiation. The spore counts of all strains were similar after growth for 9 days at $30^{\circ} \mathrm{C}$ on TBO plates. The spores of all strains were serially diluted and plated on minimal medium to check their viability,
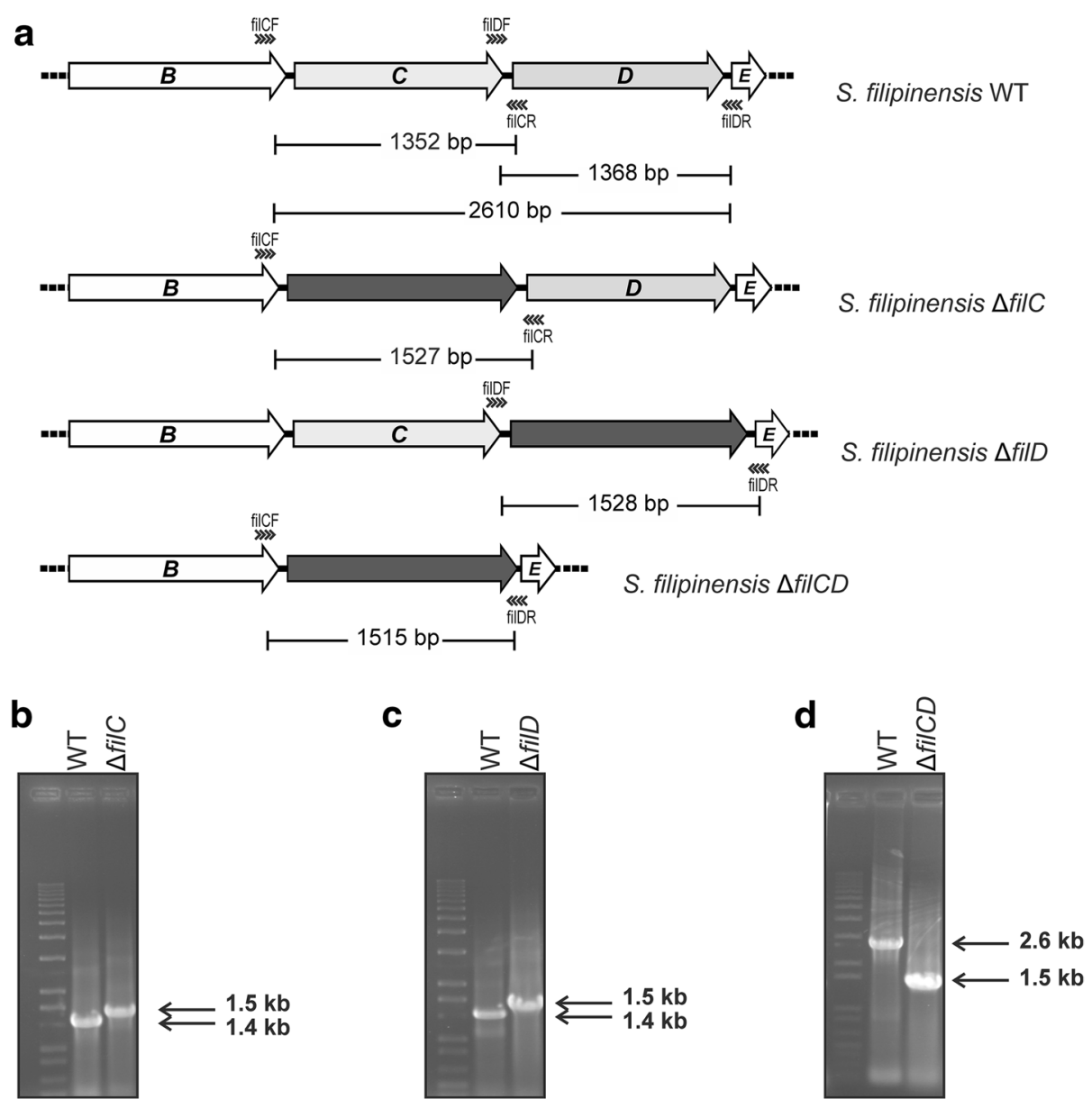

Fig. 3 Construction of cytochrome P450 mutants. a Predicted PCR fragment amplification of the parental strain and the different mutants. The primers used in the assay are indicated with arrowheads. The acc(3)/V-oriT cassette is indicated in black. b-d PCR analysis of the wild type and the mutants. 
finding no differences between them. All strains grew well in the liquid minimal medium, showing identical growth kinetics.

The fermentation broths produced by the new mutant strains, when grown in YEME medium, were extracted with ethyl acetate and analysed by thin layer chromatography for the presence of filipin III derivatives (see "Methods"). Figure 3 shows that the three mutant strains accumulated major compounds different to the filipin III produced by the parental strain, thus proving that the deleted genes were indeed filipin tailoring genes. To further characterize these compounds, we subjected extracts to high performance liquid chromatography (HPLC) analyses.

After $72 \mathrm{~h}$ of growth, S. filipinensis $\triangle \mathrm{filC}$ mutant accumulated two major products, compounds X (ca.67\%) and $\mathrm{Y}$ (ca. 33\%) (Fig. 4). Compound X was not observed in fermentation broths of the parental strain, whereas $Y$ was produced at a very low proportion (ca. 1\%). S. filipinensis $\Delta f i l D$ also produced two major products, compounds $\mathrm{Y}$ (ca. 12\%) and Z (88\%) (Fig. 4), both present in fermentation broths of the parental strain albeit at very low proportion (1 and 6\% respectively). In turn, the double mutant S. filipinensis $\triangle$ filCD accumulated a single major product $(100 \%)$ with the same retention time of compound Y (Fig. 4).

\section{Structural characterization of filipin III intermediates}

Identification of compounds $\mathrm{X}, \mathrm{Y}$ and $\mathrm{Z}$ was carried out by Mass Spectrometry (MS) followed by 1D and 2D Nuclear Magnetic Resonance (NMR) spectroscopy.

Mass Spectrometry analysis (Additional file 2: Figure S1) of compounds $\mathrm{X}, \mathrm{Y}$ and $\mathrm{Z}$ yielded lower masses than filipin III $(m / z 654.4)$, which were in agreement with the loss of one oxygen atom for compounds $X(\mathrm{~m} / z$ 638.6) and $\mathrm{Z}(\mathrm{m} / \mathrm{z} 638.5)$, and with the loss of two oxygen atoms for compound $\mathrm{Y}(\mathrm{m} / \mathrm{z} 622.5)$.

${ }^{1} \mathrm{H}$ and ${ }^{13} \mathrm{C}$-NMR data of compounds $\mathrm{X}, \mathrm{Y}$ and $\mathrm{Z}$ were virtually identical to those of filipin III. The only differences were in the resonances of the $\mathrm{C}^{\prime}{ }^{\prime}$ and $\mathrm{C} 26$ positions (Table 1). HMBC and ${ }^{1} \mathrm{H},{ }^{1} \mathrm{H}$-COSY correlations were used to assign the $\mathrm{C} 1^{\prime}\left(\delta_{\mathrm{H}}, \delta_{\mathrm{C}}\right)$ and $\mathrm{C} 26\left(\delta_{\mathrm{H}}, \delta_{\mathrm{C}}\right)$.

HMBC spectrum of compound $\mathrm{X}$ showed correlations between the signal assignable to carbonyl group at $171.68(\mathrm{C} 1)$ and the signals corresponding to $\mathrm{H}-1^{\prime}\left(\delta_{\mathrm{H}}\right.$ 3.72) and $\mathrm{H}-3\left(\delta_{\mathrm{H}} 4.00\right)$, proton chemical shifts according to hydroxylation at $\mathrm{C}^{\prime}$ and $\mathrm{C} 3 .{ }^{1} \mathrm{H},{ }^{1} \mathrm{H}-\mathrm{COSY}$ exhibited resonances of diastereotopic methylene protons $\mathrm{H}-2^{\prime}\left(\delta_{\mathrm{H}}\right.$ $\left.1.26, \delta_{\mathrm{H}} 1.31\right)$ with $\mathrm{H}-1^{\prime}$, which allowed to unambiguous assignment to $\mathrm{CH}-1^{\prime}$. The carbon chemical shifts of $\mathrm{C}-1^{\prime}$ $\left(\delta_{C} 70.47\right)$ were assigned by HSQC-HMQC spectrum. The absence of oxygenated function on $\mathrm{C} 26$ was stablished by $\mathrm{HMBC}$ correlations between $\mathrm{CH}_{3}-28\left(\delta_{\mathrm{H}} 1.17\right.$,
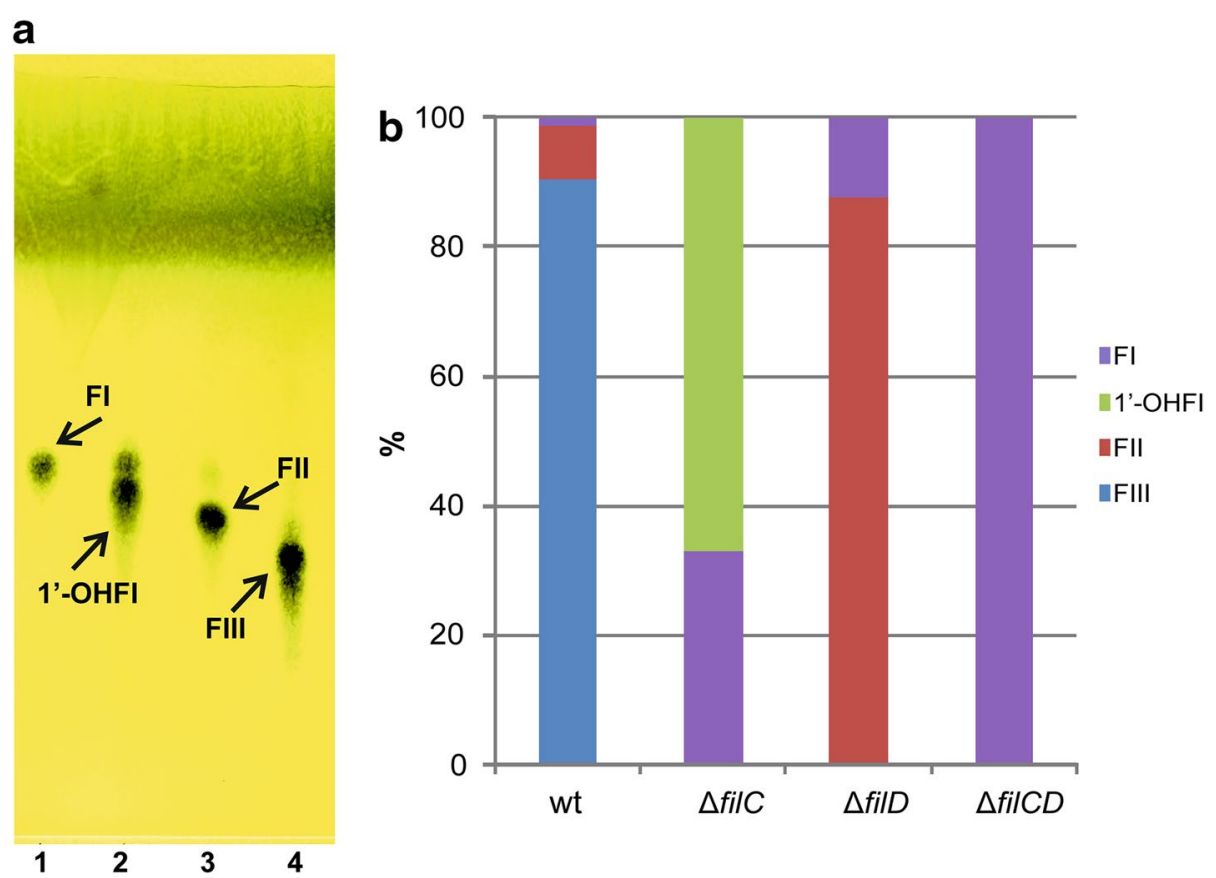

Fig. 4 Filipin intermediates generated upon gene inactivation. a TLC analyses of $72 \mathrm{~h}$ culture broth extracts of S. filipinensis (4), S. filipinensis $\Delta$ filC (2), S. filipinensis $\Delta$ filD (3), and S. filipinensis $\Delta$ filCD (1). b Relative proportions of the major filipin products accumulated by the different strains. Fl, filipin I (compound Y); Fll, filipin II (compound Z); FIII, filipin III; 1'-OHFI, 1'-hydroxyfillipin I (compound X). 
Table $1 \mathrm{MS}$ and selection of ${ }^{1} \mathrm{H}$ and ${ }^{13} \mathrm{C}$-NMR data of compounds $X, Y, Z$ and filipin III

\begin{tabular}{|c|c|c|c|}
\hline \multirow[t]{2}{*}{ Compound } & \multirow[t]{2}{*}{ MS } & \multicolumn{2}{|c|}{${ }^{1} \mathrm{H}$ and ${ }^{13} \mathrm{C}-\mathrm{NMR}$ position } \\
\hline & & 26 & $1^{\prime}$ \\
\hline X (1'-hydroxyfilipin I) & $\begin{array}{l}{[\mathrm{M}+\mathrm{Na}]^{+}} \\
661.6\end{array}$ & $\begin{array}{l}\delta_{H}: 2.24 \text { and } 2.29 \\
\delta_{C}: 37.94\end{array}$ & $\begin{array}{l}\delta_{H}: 3.72 \\
\delta_{C}: 70.47\end{array}$ \\
\hline Y (filipin I) & $\begin{array}{l}{[\mathrm{M}+\mathrm{Na}]^{+}} \\
645.5\end{array}$ & $\begin{array}{l}\delta_{H}: 2.25 \text { and } 2.35 \\
\delta_{C}: 37.89\end{array}$ & $\begin{array}{l}\delta_{H}: 1.44 \text { and } 1.68 \\
\delta_{C}: 29.47\end{array}$ \\
\hline Z (filipin II) & $\begin{array}{l}{[\mathrm{M}+\mathrm{Na}]^{+}} \\
661.5\end{array}$ & $\begin{array}{l}\delta_{H}: 3.93 \\
\delta_{C}: 72.11\end{array}$ & $\begin{array}{l}\delta_{H}: 1.43 \text { and } 1.68 \\
\delta_{C}: 29.60\end{array}$ \\
\hline Filipin III & $\begin{array}{l}{[\mathrm{M}+\mathrm{H}]^{+}} \\
655.4\end{array}$ & $\begin{array}{l}\delta_{H}: 3.94 \\
\delta_{C}: 73.00\end{array}$ & $\begin{array}{l}\delta_{H}: 3.66 \\
\delta_{C}: 69.50\end{array}$ \\
\hline
\end{tabular}

Only the chemical shifts of the most relevant signals are listed. Positions are labelled according to their number in the polyketide backbone (Fig. 6).

$\left.\delta_{\mathrm{C}} 20.31\right)$ and $\mathrm{CH}_{2}-26\left(\delta_{\mathrm{H}} 2.24\right.$ and $\left.2.29, \delta_{\mathrm{C}} 37.94\right)$. The complete assignments of NMR data are shown in Additional file 3: Table $\mathrm{S} 2\left(\delta_{\mathrm{H}}, \delta_{\mathrm{C}}, \mathrm{DEPT}\right)$ and Additional file 4 : Figure $\mathrm{S} 2\left({ }^{1} \mathrm{H},{ }^{1} \mathrm{H}-\mathrm{COSY}\right.$ and $\left.\mathrm{HMBC}\right)$.

Similarly, were identified the compound $\mathrm{Y}$ as filipin I (Table 1). The resonances of $\mathrm{C}^{\prime}\left(\delta_{\mathrm{H}} 1.44\right.$ and $1.68, \delta_{\mathrm{C}}$ $29.47)$ and $C 26\left(\delta_{\mathrm{H}} 2.25\right.$ and $\left.2.35, \delta_{\mathrm{C}} 37.89\right)$ were absent of hydroxyl group in these positions.

Finally, the compound $\mathrm{Z}$ was identified as filipin II by NMR data. The resonance of $C 1^{\prime}\left(\delta_{\mathrm{H}} 1.43\right.$ and $1.68, \delta_{\mathrm{C}}$ 29.60) was absent of hydroxyl function, while the position $\mathrm{C} 26\left(\delta_{\mathrm{H}} 3.93, \delta_{\mathrm{C}} 72.11\right)$ retained the oxygen atom.

\section{Gene complementation restores filipin III biosynthesis in the mutants}

To confirm that the gene deletions were directly responsible for the impairment on filipin III production and the accumulation of the intermediates described above, we complemented all mutants with the corresponding gene/s. For that purpose, we introduced one copy of filC into the genome of S. filipinensis $\triangle f i l C$ using the integrative plasmid pSETneo::filC (see "Methods"). pSET152neo was also introduced into S. filipinensis wild type as control. Interestingly, introduction of the vector restored completely the ability of S. filipinensis $\Delta$ filC to produce filipin III (Fig. 5). Similarly, when we introduced one copy of filD into the genome of S. filipinensis $\triangle$ filD using the integrative plasmid pSETneo::filD, or when we introduced single copies of filC and filD into the genome of S. filipinensis $\triangle$ filCD using the integrative plasmid pSETneo::filCD (see "Methods") a total restoration of filipin III production was observed (Fig. 5).

Introduction of an extra copy of filD in S. filipinensis $\Delta f i l C$, or an extra copy of filC in S. filipinensis $\Delta$ filD, did not modify their HPLC profile (not shown), thus indicating that the P450 monooxygenases they encode show strict regiospecificity, as has been demonstrated for their counterparts from S. avermitilis [14].

Strikingly, introduction of a copy of filC into the double mutant resulted in the production of filipin II, as the major product, by the recombinant strain, whereas introduction of filD into the same strain resulted in the accumulation of similar amounts of both filipin I and $1^{\prime}$-hydroxyfilipin I (Fig. 5). These results are in concordance with those observed upon inactivation of the individual genes, thus corroborating results above.

\section{There are two alternative routes for filipin III biosynthesis}

The production of filipin I as a sole product by the double mutant indicates that filipin I is the aglycone resulting from the polyketide synthases assembly line after cyclization. This is in agreement with the model proposed by Ikeda et al. [2] for S. avermitilis. In their model, filipin I is first hydroxylated at C26 to yield filipin II, and then filipin II is further hydroxylated at $\mathrm{C}^{\prime}$ to yield filipin III. The major production of filipin II (ca. 88\%) by the S. filipinensis $\Delta$ filD mutant (Fig. 4) indicates that filipin I is the substrate of FilC which hydroxylates the polyene at C26 (Fig. 6). This result somewhat demonstrates in vivo what had already been shown in vitro with recombinant CYP105P1 (PteC) that converts $50 \%$ of filipin I to filipin II [14]. Interestingly, S. filipinensis $\Delta$ filC mutant produced filipin I as expected, but also a large proportion (about 67\%) of 1'-hydroxyfilipin I (Fig. 4). This indicates that in vivo FilD is able to hydroxylate filipin $\mathrm{I}$ at $\mathrm{C1}^{\prime}$, as has been shown in vitro by recombinant CYP105D6 (PteD) [14]. These results were corroborated by gene complementation of the double mutant with filC, thus validating that FilC catalyzes the conversion of filipin I to filipin II, and with filD, confirming that FilD can convert filipin I to $1^{\prime}$-hydroxyfilipin I. Remarkably, gene complementation results also demonstrate that both filipin II and $1^{\prime}$-hydroxyfilipin I are converted to filipin III, thus indicating that there are two alternative routes for filipin III formation (Fig. 6). In one of the alternatives of the route, filipin I is converted to filipin II by FilC, and the latter is then converted to filipin III by FilD, as in the model proposed by Ikeda et al. [2]. In the second option, filipin I is first converted to $1^{\prime}$-hydroxyfilipin I by FilD, which is subsequently converted to filipin III by FilC (Fig. 6).

\section{Yield and bioactivity of filipin III intermediates}

Comparison of the amount of major products produced by the different strains indicated that the yields of polyenes in YEME medium without sucrose were very similar for the four strains, in the range of $50 \mu \mathrm{g}$ of product per mg of dry cells (around $160 \mathrm{mg} / \mathrm{l})$. In order 

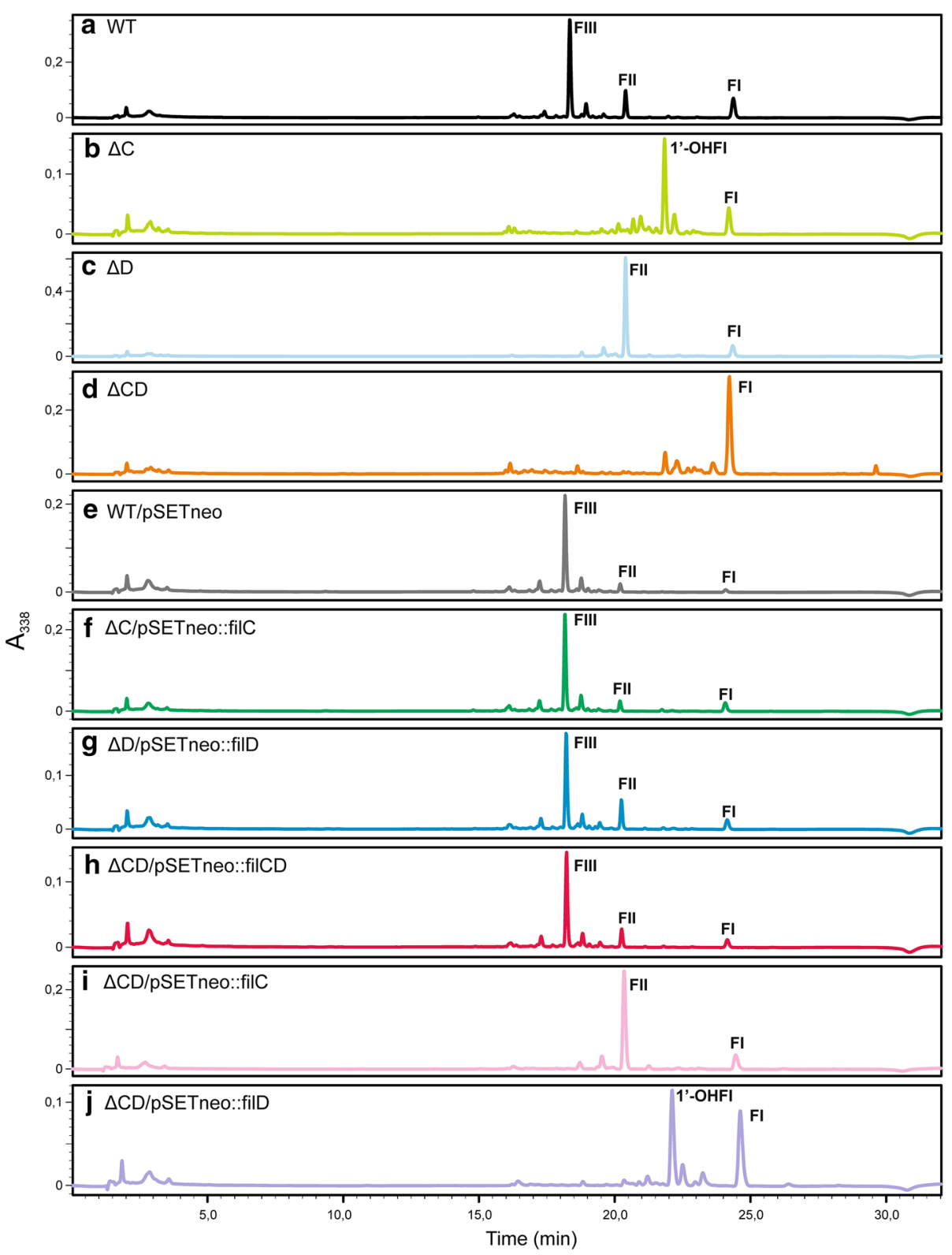

Fig. 5 Analytical HPLC of products accumulated after gene deletions and after gene complementations. Comparison of HPLC analyses of ethyl acetate-extracted broths from S. filipinensis wild-type (a), and strains S. filipinensis $\Delta$ filC (b), S. filipinensis $\Delta$ filD (c), S. filipinensis $\Delta$ filCD (d), S. filipinensis wt/pSETneo (e), S. filipinensis $\Delta$ filC/pSETneo::filC (f), S. filipinensis $\Delta$ filD/pSETneo::filD (g), S. filipinensis $\Delta$ filCD/pSETneo::filCD (h), S. filipinensis $\Delta$ filCD/ pSETneo::filC (i), and S. filipinensis $\Delta$ filCD/pSETneo:.:filD (j). Detection was carried out at $A_{338}$. Abbreviations are as in Fig. 4.

to characterise the biological activity of the products, we initially compared bioassays performed with ethyl acetate-extracted culture broths from early stationary phase-grown cells of the mutants with those of the parent strain. In the case of S. filipinensis $\Delta$ filD, we found a consistent halo of growth inhibition of the $C$. utilis cells used as test organism (Fig. 7), indicating that filipin II retains antibiotic activity. This halo was smaller than that of filipin III, thus either filipin II has lower antibiotic activity than filipin III or the compound displays a smaller diffusion rate in agar than the latter. In the case of S. filipinensis $\Delta f i l C$, we also found an halo of inhibition of Candida growth although smaller than that of filipin II, indicating that $1^{\prime}$-hydroxyfilipin I also retains antibiotic activity (Fig. 7). In turn, S. filipinensis $\triangle$ filCD yielded almost unappreciable halos under the conditions used. Similar results were observed when we used S. cerevisiae as test organism. 


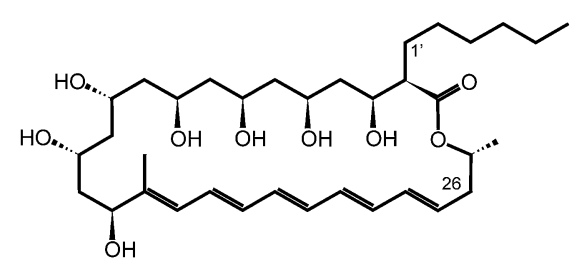

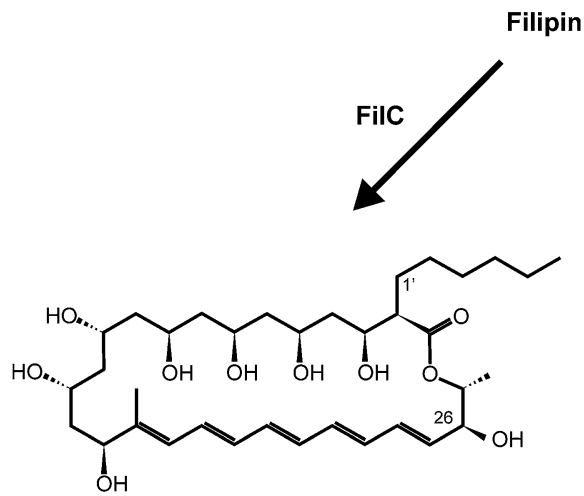

Filipin II (26-hydroxyfilipin I) $\left[\mathrm{C}_{35} \mathrm{H}_{58} \mathrm{O}_{10}\right]$
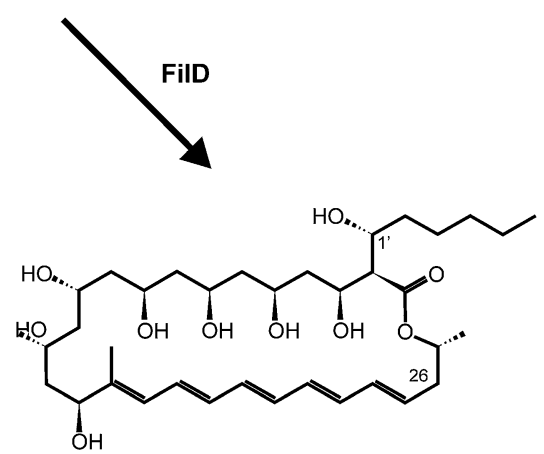

1'-Hydroxyfilipin I [ $\left.\mathrm{C}_{35} \mathrm{H}_{58} \mathrm{O}_{10}\right]$
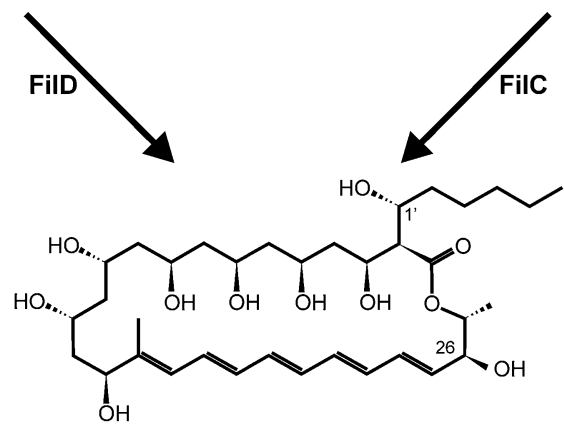

Filipin III $\left[\mathrm{C}_{35} \mathrm{H}_{58} \mathrm{O}_{11}\right]$

Fig. 6 Two alternative routes on filipin III biosynthesis in S. filipinensis.

Filipin III intermediates were then purified, and minimal inhibitory concentrations were measured by broth microdilution assay, finding MICs for C. utilis of $0.3 \mathrm{mg} / \mathrm{l}$ for filipin III and filipin I, $0.08 \mathrm{mg} / \mathrm{l}$ for $1^{\prime}$-hydroxyfilipin I, and $0.03 \mathrm{mg} / \mathrm{l}$ for filipin II (Fig. 7). S. cerevisiae turned out to be less sensitive, but the bioactivity of the compounds followed the same pattern (Fig. 7). The lower MICs of both filipin II and 1'-hydroxyfilipin I when compared with that of filipin III indicate that these new compounds show remarkably improved bioactivity against C. utilis, and given their bioassay results also suggest a lower diffusion rate than filipin III.

\section{Discussion}

The organization of the filipin tailoring genes identified in S. filipinensis turned out to be identical to that of $S$. avermitilis, except for the presence of a PadR-like encoding gene in one of the fringes of the S. filipinensis cluster that is absent in S. avermitilis. Given that this gene constitutes the main difference between both clusters, one could speculate that the enormous difference in production between both strains could be attributable to such gene. PadR belongs to a family of transcriptional regulators (Pfam PF03551), usually repressors that regulate diverse processes [24, 25, 27], although activators have also been described [26], and among those processes could be the production of the antifungal filipin. In fact, gene disruption experiments indicated that Fill is actually involved in filipin biosynthesis (Fig. 2). However, filipin production was boosted in the knocked-out mutant when compared with the parental strain, thus indicating that FilI acts as a negative regulator of the biosynthesis. 


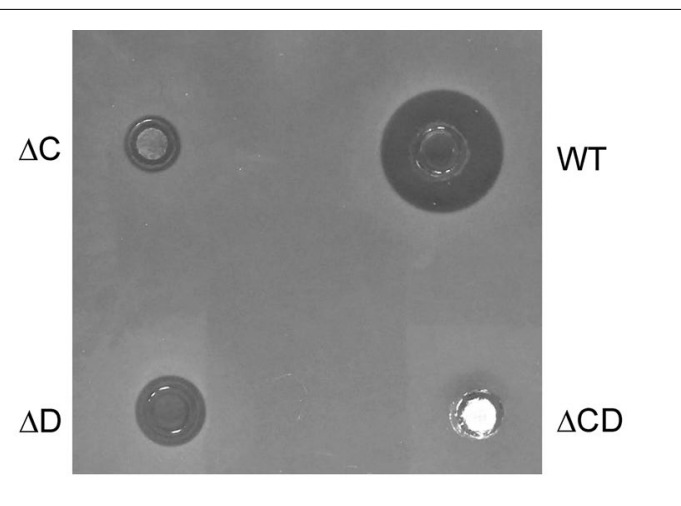

$\begin{array}{ll}\text { C. utilis } & \text { S. cerevisiae }\end{array}$

MICs $\left\{\begin{array}{lll}\text { FIII } & 0.3 \pm 0.0 \mathrm{mg} / \mathrm{l} & 0.4 \pm 0.1 \mathrm{mg} / \mathrm{l} \\ \text { FII } & 0.03 \pm 0.0 \mathrm{mg} / \mathrm{l} & 0.2 \pm 0.0 \mu \mathrm{g} / \mathrm{l} \\ 1^{\prime}-\mathrm{OHFI} & 0.08 \pm 0.0 \mathrm{mg} / \mathrm{l} & 0.3 \pm 0.1 \mathrm{mg} / \mathrm{l} \\ \mathrm{FI} & 0.3 \pm 0.1 \mathrm{mg} / \mathrm{l} & 0.4 \pm 0.1 \mathrm{mg} / \mathrm{l}\end{array}\right.$

Fig. 7 Bioassay and MICs of filipin III and its intermediates. Bioassays were performed with ethyl acetate-extracted culture broths from early stationary phase-grown cells. C. utilis was used as test organism. MICs of the purified filipin derivatives for $C$. utilis and S. cerevisiae were measured by broth microdilution assay (see "Methods"). Abbreviations are as in Fig. 4.

How a negative regulator could improve antifungal production is difficult to interpret. Fill may act either directly as a negative regulator on the promoters of given fil genes, or it may activate or repress a hierarchical regulator controlling fil genes expression, but none of these possibilities can explain the higher filipin III production of S. filipinensis, hence a different explanation must be sought. Future experimental analyses will hopefully provide an answer to this question. Noteworthy, while writing this manuscript several $S$. durhamensis sequences showing homology to fil genes have been deposited in the databases in the context of a sequencing project (GenBank: NZ_JNXR00000000.1). Those genes show the same organization as the fil genes including the padR-like regulatory gene neighboring a discrete thioesterase gene.

Besides the presence of an additional regulatory gene, the difference in polyene yield between both strains could also be explained by the changes observed in the intergenic regions that could also have effects on the gene transcription given that these regions may include promoter activities. In fact, in $S$. avermitilis there are promoters immediately upstream pteD and $p t e H$ [9], while we have not detected them in S. filipinensis. In turn, in
S. filipinensis, filF and filG belong to different transcriptional units, whereas pteG can either be co-transcribed with pteF or use its own promoter in S. avermitilis [9]. A similar situation has been described for the pimaricin producers $S$. natalensis and S. chattanoogensis that show different transcriptional organization due to changes in intergenic regions [28].

Streptomyces filipinensis mutants in P450 monooxygenase encoding genes filC and filD, and in both (filCD), were obtained by PCR-targeting. The strains so generated produced filipin III derivatives as shunt metabolites, thus establishing the link of these genes with filipin III biosynthesis. The double mutant accumulated filipin I as a sole product, whereas complementation with single copies of both filC and filD restored filipin III biosynthesis, thus demonstrating that filipin I is the direct product of PKS assembly and cyclization. The production of filipin I and 1'-hydroxyfilipin I (26-deoxyfilipin III), as major products, by S. filipinensis $\triangle \mathrm{filC}$, demonstrates that FilD is in fact the enzyme responsible for the introduction of an hydroxyl function at $\mathrm{C}^{\prime}$, whereas the production of filipin I and filipin II by S. filipinensis $\Delta$ filD, indicates that FilC is in charge of the introduction of the hydroxyl group at $\mathrm{C} 26$. Gene complementation of the mutants with a single copy of the corresponding gene restored wild-type phenotype, whereas cross-complementation was unsuccessful, indicating that both enzymes show strict regiospecificity. These results are in agreement with the in vitro results obtained with $S$. avermitilis recombinant PteC and PteD which catalyse filipin hydroxylation at $\mathrm{C} 26$ and $\mathrm{C1}^{\prime}$ respectively [14].

Notably, our results also indicate that there are two alternative routes for filipin III formation (Fig. 6). In one of the alternatives, filipin I is converted to filipin II by FilC, and the latter is then converted to filipin III by FilD, as in the model proposed by Ikeda et al. [2]. In the second option, filipin I is first converted to $1^{\prime}$-hydroxyfilipin I by FilD, which is subsequently converted to filipin III by FilC. Which one is the preferential option remains to be elucidated, although according to the complementation results of the double mutant S. filipinensis $\triangle$ filCD with the individual genes (Fig. 5), FilC seems to hydroxylate Filipin I at a higher rate than FilD. This is in agreement with the in vitro results observed with the $S$. avermitilis enzymes PteC and PteD [14]. Besides, $1^{\prime}$-hydroxyfilipin I was never observed in the wild-type strain fermentation broths, thus suggesting that FilC converts all of this intermediate to filipin III in vivo.

Identification of filipin III intermediates has been carried out by MS and various NMR techniques, and this report constitutes the first description of the complete nuclear magnetic resonance assignments for 1 '-hydroxyfilipin I. Strikingly, all filipin derivatives were 
accumulated at high yield by the mutants, which will allow the massive production of filipin III intermediates that will prove valuable as precursors for the synthetic generation of novel derivatives.

All the derivatives obtained (filipin I, filipin II, and $1^{\prime}$-hydroxyfilipin I) were biologically active. While filipin I showed a similar MIC to filipin III $(0.3$ or $0.4 \mathrm{mg} / \mathrm{l}$ depending of the test organism), the antifungal activity of 1'-hydroxyfilipin I was 3.75 or 1.33 times higher (for $C$. utilis and S. cerevisiae respectively) and that of filipin II the highest, being tenfold or twofold than that of filipin III (for C. utilis and S. cerevisiae respectively). The contrast between MICs of filipin derivatives with the sizes of the growth inhibition zones in bioassays could be attributed to the different diffusion rates of the intermediates in agar. Diffusion rate of antibiotics is a fundamental parameter of agar diffusion assays [29]. Thus, compounds having a good diffusion coefficient and low antimicrobial activity may penetrate the agar medium even in small amounts, and the reverse also holds true. Filipin III has one extra hydroxyl group when compared with filipin II or $1^{\prime}$-hydroxyfilipin I, therefore it is more polar, and its diffusion rate is higher than those of its precursors. This lack of correlation between MIC measurement and agar diffusion tests has also been reported in studies with substituted salicylaldehydes, where compounds with no activity in agar diffusion tests had potent activity in MIC tests [30], and therefore must be taken into consideration for antimicrobial activity screening. The higher antifungal activity of intermediates of the pathway was unexpected given that, normally, the final product is the compound with greater bioactivity $[31,32]$. But considering that filipin III shows a similar affinity for cholesterol and ergosterol [33], it is likely that both filipin II and $1^{\prime}$-hydroxyfilipin I show an improved affinity for ergosterol than filipin III. Future analysis of other parameters important for the applicability of these designer polyenes such as host-range, toxicity, and acid or alkali stability will establish the pharmaceutical importance of the findings presented here.

\section{Conclusions}

Genetic manipulations of polyene biosynthetic pathways have proven useful for the discovery of products with improved properties. Here, we describe the late biosynthetic steps for the biosynthesis of the pentaene macrolide filipin III, finding that two alternative routes lead to its formation. In one of the routes, filipin I is converted to filipin III via filipin II, whereas in the other, the intermediate is $1^{\prime}$-hydroxyfilipin I, an unnatural compound. All intermediates were produced at high yield, and showed antifungal activity, being $1^{\prime}$-hydroxyfilipin and especially filipin II remarkably more active than filipin III. These results open new possibilities for the generation of biologically active filipin derivatives at high yield and with improved properties.

\section{Methods}

\section{Bacterial strains and cultivation}

Streptomyces filipinensis DSM 40112 was used as the source of DNA in the construction of the genomic library, and was routinely grown in YEME medium [34] without sucrose. Sporulation was achieved as described elsewhere [35]. Escherichia coli strain DH5 $\alpha$ was used as a host for DNA manipulation. E. coli strain XL1-Blue MR was used for obtaining SuperCos 1 cosmid (Stratagene) recombinant derivatives. E. coli BW25113 [pIJ790] was used for gene replacement experiments. E. coli ET12567 [pUZ8002] was used as donor in intergeneric conjugations. C. utilis (syn. Pichia jadinii) CECT 1061 and S. cerevisiae CECT 1942 were used for bioassay experiments. Minimum inhibitory concentrations (MICs) were determined by the broth microdilution technique following the EUCAST methodology by diluting filipin III derivatives in RPMI 1640 with glutamine and $0.2 \%$ glucose but without sodium bicarbonate (Sigma) buffered with $0.164 \mathrm{M}$ MOPS pH 7.0 to concentrations of $40 \mu \mathrm{g} /$ $\mathrm{ml}$ of which $100 \mu \mathrm{l}$ was added to the first row of a 96-well suspension culture plate. This was followed by a 1:1 dilution series in medium. The plates were spotted with 2,000 viable $C$. utilis or S. cerevisiae cells. The MIC value was determined to be the lowest concentration of antibiotic, which inhibited the growth of the yeast strain and could be determined by eye on the 96-well plate after an incubation of $24 \mathrm{~h}$ at $30^{\circ} \mathrm{C}$. Commercial filipin III (Sigma) was used as control.

\section{Plasmids and DNA manipulation procedures}

Standard genetic techniques with $E$. coli and in vitro DNA manipulations were as described by Sambrook and Russell [36]. Recombinant DNA techniques in Streptomyces species and isolation of Streptomyces total DNA were performed as previously described [34]. For construction of the genomic library, S. filipinensis genomic DNA was partially digested with Sau3AI and fragments in the $35 \pm 40 \mathrm{~kb}$ size range were cloned into SuperCos 1 digested with BamHI and XbaI. The ligation mixture was packaged with Gigapack III XL (Stratagene) and used to transfect E. coli XL-1 Blue MR. The library was screened using probes obtained by PCR amplification of S. filipinensis chromosomal DNA by using oligonucleotides derived from conserved stretches of the filipin PAS-LuxR regulator pteF from S. avermitilis [9]. The oligonucleotide pairs used were 
5'-CGGCTCGTCCGAGGACATATGC-3' ${ }^{\prime}$ and $5^{\prime}$-AAT CACGCTGTGGCTCCTGAGCTCGGG-3'. A positive clone (Cos 8G9) containing a pteF homologous gene was used as the starting point for chromosome walking. Southern hybridization was carried out with probes labelled with digoxigenin by using the DIG DNA labelling kit (Roche Biochemicals). Intergeneric conjugation between E. coli ET12567 [pUZ8002] and S. filipinensis was performed as described [37]. pUC19 (New England Biolabs) was used as the routine cloning vector, pSETneo $\left(\mathrm{Am}^{\mathrm{R}}, \mathrm{Kan}^{\mathrm{R}}\right.$, pUC18 replicon, $\Phi C 31$ attP; [38]), was used for intergeneric conjugation. Polymerase chain reactions were carried out using Phusion DNA polymerase as described by the enzyme supplier (Finnzymes). DNA sequencing was accomplished by the dideoxynucleotide chain-termination method using the Perkin Elmer Amplitaq Gold Big Dye-terminator sequencing system with an Applied Biosystems ABI 3130 DNA genetic analyzer (Foster City, CA, USA).

\section{RNA isolation and reverse transcriptase-PCR experiments}

RNA was isolated as described [9]. Transcription was studied by using the SuperScript ${ }^{\mathrm{TM}}$ One-Step reverse transcriptase-PCR (RT-PCR) system with Platinum ${ }^{\circledR}$ Taq DNA polymerase (Invitrogen), using $200 \mathrm{ng}$ of total RNA as template. Conditions were as follows: first strand complementary DNA (cDNA) synthesis, $50^{\circ} \mathrm{C}$ for $40 \mathrm{~min}$ followed by heating at $94^{\circ} \mathrm{C}$ for $2 \mathrm{~min}$; amplification, 40 cycles of $98^{\circ} \mathrm{C}$ for $15 \mathrm{~s}, 59-68^{\circ} \mathrm{C}$ (depending of the set of primers used) for $30 \mathrm{~s}$, and $72^{\circ} \mathrm{C}$ for $1 \mathrm{~min}$. Primers (18-28 mers; Additional file 1 : Table S1) were designed to cover intergenic regions, generating PCR products of approximately 100$650 \mathrm{bp}$. Negative controls were carried out with each set of primers and Platinum ${ }^{\circledR}$ Taq DNA polymerase in order to confirm the absence of contaminating DNA in the RNA preparations. The identity of each amplified product was corroborated by direct sequencing of the PCR product.

\section{Construction of $\Delta$ filC, $\Delta$ filD, $\Delta$ filCD, and $\Delta$ fill mutants}

Deletion of filC from S. filipinensis chromosome was made by replacing the wild-type gene with a cassette containing an apramycin selective marker using a PCR based system [39]. The plasmid pIJ773 containing the apramycin resistance gene $(a a c(3) I V)$ and the oriT replication origin was used as a template. The mutant was constructed using the oligonucleotides (CDF) 5'-gtacgacccccccacccacaa gctccaaggagagccatgATTCCGGGGATCCGTCGACC-3' and (CR) 5'-gggcggtgcgggcgtgcgtcgagatgtgaggccgggt caTGTAGGCTGGAGCTGCTTC- $3^{\prime}$ as the forward and reverse primers respectively (the sequence identical to the DNA segment upstream from the start codon of
filC is in lower case italics and the sequence identical to the segment downstream from the stop codon of filC is underlined and in lower case). These two long PCR primers (59 and $58 \mathrm{nt}$ ) were designed to produce a deletion of filC just after its start codon leaving only its stop codon behind. The $3^{\prime}$ sequence of each primer matches the right or left end of the disruption cassette (the sequence is shown uppercase in both primers). The extended resistance cassette was amplified by PCR and E. coli BW25113/ pIJ790 bearing cosmid $8 \mathrm{C} 8$ was electro-transformed with this cassette. The isolated mutant cosmid was introduced into non-methylating $E$. coli ET12567 containing the RP4 derivative pUZ8002. The mutant cosmid was then transferred to $S$. filipinensis by intergeneric conjugation. Double cross-over exconjugants were screened for their apramycin resistance followed by confirmation by PCR.

Mutant $\triangle$ filD was constructed following the same strategy, using the oligonucleotides (DF) 5'-gcacgcccg caccgcccttgctcgaaaggcaccacatgTGTAGGCTGGAGCTG CTTC-3' and (CDR) 5'-ccgccaccctttccttccgttccgtccg gccgggccgtcaATTCCGGGGATCCGTCGACC-3' as the forward and reverse primers respectively, and the same cosmid template. Similarly, mutant $\triangle$ filCD was constructed using oligonucleotides $\mathrm{CDF}$ and CDR. Mutant $\triangle$ fill was constructed using oligonucleotides (IF) 5'-ttgctatgcaacgagttgcatagcaggatcgacggcatgATTCCGGGGATCCGTCGACC-3' and (IR) $5^{\prime}$-ctggaccggcgggacctgcgggcggtacggcggcgatcaTGTAG GCTGGAGCTGCTTC-3', and cosmid 8G9 as template.

In all cases, mutants were verified by PCR analysis (Figs. 2, 3).

\section{Construction of plasmids for gene complementation}

In order to complement filC replacement mutant, a $1,352 \mathrm{bp}$ DNA fragment containing the entire filC gene plus its upstream intergenic region was amplified by PCR with primers filCF (5'-GGAATTCGCTCGCCGCCGCC TGAC-3') and filCR (5'-GGAATTCGGCGGATGTCGG TGTCGGTC-3') using S. filipinensis chromosomal DNA as template. The PCR product was cloned into an EcoRIcut pSETneo, and the construction with the gene in the same orientation of the neo gene was selected to yield pSETneo::filC. This would permit gene expression driven from the promoter of the neo gene in case the cloned DNA fragment lacked promoter activity.

Similarly, for S. filipinensis $\Delta$ filD gene complementation, a 1,368 bp DNA fragment containing the entire filD gene plus its upstream intergenic region was amplified by PCR with primers filDF (5'-GGAATTCGAGCTGCC CGTCACCTGG-3') and filDR (5'-GGAATTCGCGG TCGATGGTGATGCG-3'). The PCR product was cloned into an EcoRI-cut pSETneo to yield pSETneo::filD after selection of gene orientation. 
Similarly, for the complementation of the double mutant, a $2.6 \mathrm{~kb}$ DNA fragment containing filC and filD was amplified by PCR with primers filCF and filDR. The PCR product was then cloned into the EcoRI site of pSETneo to yield pSETneo::filCD.

\section{Assay of filipin production}

To determine filipins production and metabolite purification, one volume of culture was extracted with two volumes of ethyl acetate, and the organic phase was sequentially treated with saturated $\mathrm{NaCl}$ solution and $\mathrm{Na}_{2} \mathrm{SO}_{4}$, vacuum dried, and resuspended in pure methanol. For routine determination of metabolite yield, cultures were extracted with one volume of methanol, and diluted when needed. Solutions of pure filipin III (Sigma) were used as control. Quantitative determination of filipins was assessed by reverse phase HPLC using a Waters 600 unit coupled to a diode array ultraviolet detector set at $338 \mathrm{~nm}$ equipped with a Mediterranean Sea C18 column $(4.6 \times 150 \mathrm{~mm}$, particle size $3 \mu \mathrm{m}$ ) (Teknokroma). Elution was performed with a gradient $(0.8 \mathrm{ml} / \mathrm{min})$ of methanol- $0.1 \%$ formic acid according to the following program (50:50 $\mathrm{v} / \mathrm{v} \mathrm{0}$-3 min, up to $90: 10 \mathrm{v} / \mathrm{v}$ 3-12 $\mathrm{min}, 90: 10$ 12-20 min, up to $100: 0 \mathrm{v} / \mathrm{v} 20-21 \mathrm{~min}, 100: 0 \mathrm{v} / \mathrm{v} 21-23 \mathrm{~min}$, down to 0:100 v/v 23-24 min, 0:100 v/v 24-26 min, up to $50: 50 \mathrm{v} / \mathrm{v}$ 26-27 min, 50:50 v/v 27-32 min). Retention time for Filipin III was $18.2 \mathrm{~min}$. Thin layer chromatography was performed on silica 60 F254 plates (Merck), and elution was carried out with dichloromethane:methanol (4:1) (v/v). Plates were developed by spraying with phosphomolybdic acid:ethanol (1:9) $(\mathrm{v} / \mathrm{v})$ and heating.

\section{Structural elucidation of $\mathbf{1}^{\prime}$-hydroxyfilipin I}

Fermentation broths of $S$. filipinensis $\triangle$ filC were harvested by centrifugation after 3 days of growth at $250 \mathrm{rpm}$ and $30^{\circ} \mathrm{C}$, and the supernatant treated as indicated above. For MS analyses, the dry residue was dissolved in methanol, whereas for nuclear magnetic resonance (NMR) determination, the dry residue was dissolved in deuterated dimethyl sulfoxide.

Mass spectra experiments were taken on an Ultrafex III MALDI TOF/TOF apparatus (Bruker) by using dithranol $+\mathrm{NaI}$ as the matrix and FAB-MS spectrum was recorded on a VG Autospectrum (Waters) instrument using $m$-nitrobenzyl alcohol ( $m$-NBA) as the matrix and Caesium $\left(\mathrm{Cs}^{+}\right)$as ion bombardment at $35 \mathrm{kV}$. A filipin III solution $(5 \mathrm{mg} / \mathrm{ml}$ methanol) was used for tuning.

The structure was elucidated on the basis of the 1D-NMR: ${ }^{1} \mathrm{H}-\mathrm{NMR},{ }^{13} \mathrm{C}-\mathrm{NMR}$ and DEPT (Distortionless Enhancement by Polarization Transfer), and 2D-NMR: COSY (H,H-Correlation Spectroscopy), HSQC (Heteronuclear Single Quantum Coherence), HMQC (Heteronuclear Multiple Quantum Correlation), and HMBC
(Heteronuclear Multiple Bond Correlation) experiments, and by comparison with the spectrum of filipin III [40]. NMR spectra were recorded in DMSO- $d_{6}$ at room temperature using a Bruker WM500 spectrometer $[500 \mathrm{MHz}$ $\left({ }^{1} \mathrm{H}-\mathrm{NMR}\right)$ and $\left.125 \mathrm{MHz}\left({ }^{13} \mathrm{C}-\mathrm{NMR}\right)\right]$. The pulse programmes of the two-dimensional experiments were taken from the Bruker software library, and the parameters were as follows: $500 / 125 \mathrm{MHz}$ gradient-selected HMQC spectra: relaxation delay $D 1=1.5 \mathrm{~s} ; 500 / 125 \mathrm{MHz}$ gradient-selected $\mathrm{HMBC}$ spectra: relaxation delay $D 1=1.5 \mathrm{~s}$, evolution delay $D 2=3.33 \mathrm{~ms}$; delay for evolution of longrange coupling $D 6=60 \mathrm{~ms}$. $500 \mathrm{MHz}$ gradient-selected ${ }^{1} \mathrm{H},{ }^{1} \mathrm{H}$ COSY spectra: relaxation delay D1 $=1.5 \mathrm{~s} ; 90^{\circ}$ pulse for ${ }^{1} \mathrm{H}$.

\section{Accession number}

The sequence has been deposited in the GenBank database under the accession number KP769541.

\section{Additional files}

Additional file 1: Table S1. Primers used in RT-PCR experiments.

Additional file 2: Figure S1. MS identification of filipin intermediates. A) MALDI TOF/TOF spectrum showing the molecular ion MNa+ (645.5) of compound $Y$ (filipin I). B) MALDI TOF/TOF spectrum of compound Z (filipin II) ( $\mathrm{MNa}+=661.5)$. C) FAB spectrum of compound X (1'-hydroxyfilipin I) showing the molecular ion $\mathrm{MNa}+$ (661.6) of the complete molecule.

Additional file 3: Table S2. ${ }^{1} \mathrm{H}$ and ${ }^{13} \mathrm{C}$ - NMR chemical shifts assignments for 1 '-hydroxyfilipin I and Filipin III.

Additional file 4: Figure S2. ${ }^{1} \mathrm{H},{ }^{1} \mathrm{H}-\mathrm{COSY}$ and $\mathrm{HMBC}$ correlations of 1 '-hydroxyfilipin I.

\section{Authors' contributions}

TDP, CMV and JFA designed the research; TDP, CMV, AR, EGB, JSA, and AdP performed the research; TDP, EGB and JFA analysed the data; JFA wrote, and all the authors corrected and approved, the paper. All authors read and approved the final manuscript.

\section{Author details}

${ }^{1}$ Area of Microbiology, Faculty of Biology, Universidad de León, 24071 León, Spain. ${ }^{2}$ Institute of Biotechnology INBIOTEC, Parque Científico de León, Avda. Real, no 1, 24006 León, Spain. ${ }^{3}$ Department of Organic Chemistry, Faculty of Sciences, Campus de Cantoblanco, Universidad Autónoma de Madrid, 28049 Madrid, Spain. ${ }^{4}$ Present Address: Department of Molecular Microbiology, John Innes Centre, Norwich Research Park, Norwich NR4 7UH, UK.

\section{Acknowledgements}

This work was supported by the Spanish Ministerio de Economía y Competitividad (Grants BIO2010-19911 and BIO2013-42983-P to JFA), F.P.U. fellowships of the Ministerio de Educación, Cultura y Deporte (AP2005-3644 to JSA, AP2007-02055 to TDP, FPU13/01537 to AP), a contract from the Junta de Castilla y León co-financed by the European Social Fund (to EGB), and a fellowship from the Portuguese Fundação para a Ciência e a Tecnologia (SFRH/ BD/64006/2009 to (MV).

\section{Compliance with ethical guidelines}

Competing interests

The authors declare that they have no competing interests. 
Received: 25 May 2015 Accepted: 28 July 2015

Published online: 07 August 2015

\section{References}

1. Ammann A, Gottlieb D, Brock TD, Carter HE, Whitfield GB (1955) Filipin, an antibiotic effective against fungi. Phytopathology 46:559-562

2. Ikeda H, Shin-ya K, Omura S (2014) Genome mining of the Streptomyces avermitilis genome and development of genome-minimized hosts for heterologous expression of biosynthetic gene clusters. J Ind Microbiol Biotechnol 41:233-250

3. Kim JD, Han JW, Hwang IC, Lee D, Kim BS (2012) Identification and biocontrol efficacy of Streptomyces miharaensis producing filipin III against Fusarium wilt. J Basic Microbiol 52:150-159

4. Aparicio JF, Mendes MV, Antón N, Recio E, Martín JF (2004) Polyene macrolide antibiotic biosynthesis. Curr Med Chem 11:1645-1656

5. Kruth HS, Comlyg ME, Butlerll JD, Vanierl MT, Finks JK, Wenger DA et al (1986) Type C Niemann-Pick disease: abnormal metabolism of low density lipoprotein in homozygous and heterozygous fibroblasts. J Biol Chem 261:16769-16774

6. Gimpl G, Gehrig-Burger K (2011) Probes for studying cholesterol binding and cell biology. Steroids 76:216-231

7. Omura S, Ikeda H, Ishikawa J, Hanamoto A, Takahashi C, Shinose M et al (2001) Genome sequence of an industrial microorganism Streptomyces avermitilis: deducing the ability of producing secondary metabolites. Proc Natl Acad Sci USA 98:12215-12220

8. Ikeda H, Ishikawa J, Hanamoto A, Shinose M, Kikuchi H, Shiba T et al (2003) Complete genome sequence and comparative analysis of the industrial microorganism Streptomyces avermitilis. Nat Biotechnol 21:526-531

9. Vicente CM, Santos-Aberturas J, Payero TD, Barreales EG, de Pedro A, Aparicio JF (2014) PAS-LuxR transcriptional control of filipin biosynthesis in S. avermitilis. Appl Microbiol Biotechnol 98:9311-9324

10. Bergy ME, Eble TE (1968) The filipin complex. Biochemistry 7:653-659

11. Edwards DM (1989) Direct liquid introduction LC-MS of polyene macrolide antibiotics: comment on the structure of filipin II. J Antibiot 42:322-324

12. Pandey RC, Rinehart KL (1970) Polyene antibiotics V. Characterization of components of the filipin complex by mass spectrometry. J Antibiot 23:414-417

13. Volpon $L$, Lancelin $L$ (2000) Solution NMR structures of the polyene macrolide antibiotic filipin III. FEBS Lett 478:137-140

14. Xu LH, Fushinobu S, Takamatsu S, Wakagi T, Ikeda K, Shoun H (2010) Regio- and stereospecificity of filipin hydroxylation sites revealed by crystal structures of cytochrome P450 105P1 and 105D6 from Streptomyces avermitilis. J Biol Chem 285:16844-16853

15. Yoo HG, Kwon SY, Kim S, Karki S, Park ZY, Kwon HJ (2011) Characterization of 2-octenoyl-CoA carboxylase/reductase utilizing pteB from Streptomyces avermitilis. Biosci Biotechnol Biochem 75:1191-1193

16. Antón N, Mendes MV, Martín JF, Aparicio JF (2004) Identification of PimR as a positive regulator of pimaricin biosynthesis in Streptomyces natalensis. J Bacteriol 186:2567-2575

17. Santos-Aberturas J, Vicente CM, Payero TD, Martín-Sánchez L, Cañibano C, Martín JF et al (2012) Hierarchical control on polyene macrolide biosynthesis: PimR modulates pimaricin production via the PAS-LuxR transcriptional activator PimM. PLoS One 7:e38536

18. Santos-Aberturas J, Payero TD, Vicente CM, Guerra SM, Cañibano C, Martín JF et al (2011) Functional conservation of PAS-LuxR transcriptional regulators in polyene macrolide biosynthesis. Metab Eng 13:756-767

19. Santos-Aberturas J, Vicente CM, Guerra SM, Payero TD, Martín JF, Aparicio JF (2011) Molecular control of polyene macrolide biosynthesis: direct binding of the regulator PimM to eight promoters of pimaricin genes and identification of binding boxes. J Biol Chem 286:9150-9161

20. Vicente CM, Payero TD, Santos-Aberturas J, Barreales EG, de Pedro A, Aparicio JF (2015) Pathway-specific regulation revisited: cross-regulation of multiple disparate gene clusters by PAS-LuxR transcriptional regulators. Appl Microbiol Biotechnol 99:5123-5135

21. Aparicio JF, Martín JF (2008) Microbial cholesterol oxidases: bioconversion enzymes or signal proteins? Mol BioSyst 4:804-809
22. Heathcote ML, Staunton J, Leadlay PF (2001) Role of type II thioesterases: evidence for removal of short acyl chains produced by aberrant decarboxylation of chain extender units. Chem Biol 8:207-220

23. Kim BS, Cropp TA, Beck BJ, Sherman DH, Reynolds KA (2002) Biochemical evidence for an editing role of thioesterase II in the biosynthesis of the polyketide pikromycin. J Biol Chem 277:48028-48034

24. Gury J, Barthelmebs L, Tran NP, Diviès C, Cavin JF (2004) Cloning, deletion, and characterization of PadR, the transcriptional repressor of the phenolic acid decarboxylase-encoding padA gene of Lactobacillus plantarum. Appl Environ Microbiol 70:2146-2153

25. Fibriansah G, Kovács ÁT, Pool TJ, Boonstra M, Kuipers OP, Thunnissen AM (2012) Crystal structures of two transcriptional regulators from Bacillus cereus define the conserved structural features of a PadR subfamily. PLoS One 7:e48015

26. Flórez AB, Alvarez S, Zabala D, Braña AF, Salas JA, Méndez C (2014) Transcriptional regulation of mithramycin biosynthesis in Streptomyces argillaceus: dual role as activator and repressor of the PadR-like regulator MtrY. Microbiology 161:272-284

27. Madoori PK, Agustiandari H, Driessen AJ, Thunnissen AM (2009) Structure of the transcriptional regulator $L m r R$ and its mechanism of multidrug recognition. EMBO J 28:156-166

28. Du YL, Li SZ, Zhou Z, Chen SF, Fan WM, Li YQ (2011) The pleiotropic regulator AdpAch is required for natamycin biosynthesis and morphological differentiation in Streptomyces chattanoogensis. Microbiology 157:1300-1311

29. Bonev B, Hooper J, Parisot J (2008) Principles of assessing bacterial susceptibility to antibiotics using the agar diffusion method. J Antimicrob Chemother 61:1295-1301

30. Pelttari E, Lehtinen M, Elo H (2011) Substituted salicylaldehydes as potential antimicrobial drugs: minimal inhibitory and microbicidal concentrations. Z Naturforsch 66c:571-580

31. Mendes MV, Recio E, Fouces R, Luiten R, Martin JF, Aparicio JF (2001) Engineered biosynthesis of novel polyenes: a pimaricin derivative produced by targeted gene disruption in Streptomyces natalensis. Chem Biol 8:635-644

32. Santos-Aberturas J, Engel J, Dickerhoff J, Dorr M, Rudroff F, Weisz K et al (2014) Exploration of the substrate promiscuity of biosynthetic tailoring enzymes as a new source of structural diversity for polyene macrolide antifungals. ChemCatChem 7:490-500

33. te Welscher YM, ten Napel HH, Balagué MM, Souza CM, Riezman H, de Kruijff B et al (2008) Natamycin blocks fungal growth by binding specifically to ergosterol without permeabilizing the membrane. J Biol Chem 283:6393-6401

34. Kieser T, Bibb MJ, Buttner MJ, Chater KF, Hopwood DA (2000) Practical Streptomyces genetics. John Innes Foundation, Norwich

35. Recio E, Aparicio JF, Rumbero A, Martín JF (2006) Glycerol, ethyleneglycol and propanediol elicit pimaricin biosynthesis in the Pl-factor defective strain Streptomyces natalensis npi287 and increase polyene production in several wild type actinomycetes. Microbiology 152:3147-3156

36. Sambrook J, Russell DW (2001) Molecular cloning: a laboratory manual, 3rd edn. Cold Spring Harbor Laboratory Press, New York

37. Enríquez LL, Mendes MV, Antón N, Tunca S, Guerra SM, Martín JF et al (2006) An efficient gene transfer system for the pimaricin producer Streptomyces natalensis. FEMS Microbiol Lett 257:312-318

38. Vicente CM, Santos-Aberturas J, Guerra SM, Payero TD, Martín JF, Aparicio JF (2009) PimT, an amino acid exporter controls polyene production via secretion of the quorum sensing pimaricin-inducer PI-factor in Streptomyces natalensis. Microb Cell Fact 8:33

39. Gust B, Challis GL, Fowler K, Kieser T, Chater KF (2003) PCR-targeted Streptomyces gene replacement identifies a protein domain needed for biosynthesis of the sesquiterpene soil odor geosmin. Proc Natl Acad Sci USA 100:1541-1546

40. Rochet $\mathrm{P}$, Lancelin J (1997) Revised ${ }^{1} \mathrm{H}$ and ${ }^{13} \mathrm{C}$ NMR assignments of the polyene antibiotic filipin III. Magn Reson Chem 35:538-542 